\section{Arritmias en la distrofia miotónica de Steinert}

Queremos felicitar a Rosado A. y Sierra L. por su artículo" "Distrofia miotónica de Steinert" y nos gustaría aprovechar la oportunidad para resaltar algunos aspectos cardiológicos de la enfermedad, ya que la afectación cardiológica juega un papel destacado en las patologías neuromusculares hereditarias y en especial en la distrofia miotónica de Steinert (DMS).

La enfermedad cardiaca suele ser la responsable de la disminución de la expectativa de vida en los pacientes con $\mathrm{DMS}^{2}$. La edad media de fallecimiento en la DMS está en torno a los 50 años, el $20 \%$ se debe a causas cardiacas, de las cuales la muerte súbita representa el $10 \%{ }^{3}$. Habitualmente la muerte súbita se debe a la aparición de arritmias ventriculares o al bloqueo aurículo-ventricular completo con asistolia. Por este motivo, en los pacientes sintomáticos se debe plantear la colocación de un desfibrilador automático implantable $o$ de un marcapasos definitivo, no solo como prevención secundaria sino también como prevención primaria ${ }^{4}$.

Las alteraciones electrocardiográficas más frecuentes de la DMS están relacionadas con alteraciones en la generación o propagación del impulso cardiaco, como puede ser el bloqueo aurículo-ventricular y los bloqueos intraventriculares. Además, se observa con frecuencia la prolongación del intervalo QTc y una gran variedad de taquiarritmias. Entre las arritmias supraventriculares destacan la taquicardia, el aleteo y la fibrilación auricular, presentes en el $25 \%$ de los casos. Dentro de las arritmias ventriculares se incluyen las extrasístoles ventriculares, las taquicardias ventriculares monoformas $y$ polimorfas, la torsade de pointes y la fibrilación ventricular ${ }^{2}$.
Es recomendable la realización de un electrocardiograma anual en los pacientes con DMS para detectar, en pacientes asintomáticos, la existencia de alteraciones en la conducción cardiaca, incluso algunos autores ${ }^{2}$ han sugerido la realización de un electrocardiograma Holter en estos pacientes. Los pacientes asintomáticos con ECG anormal 0 aquellos que presenten palpitaciones o síncope, deben ser derivados para valoración por cardiología ${ }^{5}$.

\section{BIBLIOGRAFÍA}

1. Rosado A, Sierra L. Distrofia miotónica de Steinert. Rev Clin Med Fam. 2015;8(1):79-83.

2. Pelargonio G, Dello Russo A, Sanna T, De Martino G, Bellocci F. Myotonic dystrophy and the heart. Heart. 2002;88:665-70.

3. Mathieu J, Allard P, Potvin L, Prevost C, Begin P. A 10year study of mortality in a cohort of patients with myotonic dystrophy. Neurology. 1999;52:1658-62.

4. Pujol VA, Kauffman A. Cardiopatías y arritmias en patologías neuromusculares hereditarias. Un desafío diagnóstico para el cardiólogo. Rev Electro y Arritmias. 2010;2:55-62.

5. Scottish Muscle Network Managed Clinical Network. Management of Adults with Myotonic Dystrophy. A Guide for Professionals [Internet]. NHS; 2009 julio [citado 21 de Julio 2015]. Disponible en: http://www.smn.scot. nhs.uk/documents/DM1\%20PROFESSIONALS\%20 LEAFLETUpdated\%20June10. pdf.

\section{Pedro Gargantilla Maderaa, , Noelia Arroyo Pardo $^{c}$ y Emilio Pintor Holguín ${ }^{b}$}

aServicio de Medicina Interna. Hospital de EI Escorial (Madrid). bUniversidad Europea de Madrid.

'Residente de Medicina de Familia y Comunitaria. Hospital de El Escorial (Madrid).

Correo electrónico: pgargantilla@yahoo.es 